\title{
Arzthaftung bei delegierbaren Leistungen
}

Volker Großkopf, Erika Mendoza

\section{Juristische Themen sind sicher nicht der Fokus unseres täglichen Handelns in den Arztpraxen. Damit haben Ärzte in der Regel selten etwas zu tun, und wenn doch, kümmern sich darum die Haftpflichtversicherung und die Rechtsschutzversicherung.}

\begin{tabular}{|c|c|}
\hline \multicolumn{2}{|c|}{ ABKÜRZUNGEN } \\
\hline Az. & Aktenzeichen \\
\hline BGB & Bürgerliches Gesetzbuch \\
\hline CME & Continuing Medical Education \\
\hline MFA & medizinische Fachangestellte \\
\hline MTA & medizinisch-technische Assistentin \\
\hline MTAG & $\begin{array}{l}\text { Gesetz über technische Assistenten } \\
\text { in der Medizin* }\end{array}$ \\
\hline MTRA & $\begin{array}{l}\text { medizinisch-technische Radiologieassis- } \\
\text { tentin }\end{array}$ \\
\hline PfIBG & Pflegeberufsgesetz \\
\hline QM & Qualitätsmanagement \\
\hline SG & Sozialgericht \\
\hline SGB V & 5. Sozialgesetzbuch \\
\hline TFG & Transfusionsgesetz \\
\hline VERAH & $\begin{array}{l}\text { Versorgungsassistentin in der Hausarzt- } \\
\text { praxis }\end{array}$ \\
\hline
\end{tabular}

\section{Einleitung}

Die Fülle der Anforderungen, die sowohl in einer Arztpraxis als auch in einer Klinik an die handelnden Ärzte gestellt werden, geht weit über das medizinische Handlungsfeld hinaus. Neben dem Erwerb der so wichtigen CME-Weiterbildungspunkte, um nachzuweisen, dass man sein Wissen auf dem anerkannten Stand der medizinischen Wissenschaft gehalten hat, kommen Strahlenschutzkurse oder andere Eignungskurse, Nachweise für neue Techniken, Kontrolle der Weiterbildungen der Mitarbeiter sowie die Förderung und Aktualisierung von deren Wissens- und Kenntnisstand.

Das leidige Thema Praxis-Qualitätsmanagement (QM) mit all den Verfahrensanordnungen, Ausführungsanweisungen, hinterlegten Leitlinien etc. wird gern delegiert, wobei diese „Delegation“ nicht die ist, auf die sich der aktuelle Beitrag bezieht. Tatsächlich ist das QM die Niederschrift des Selbstverständlichen.

\begin{abstract}
Merke
Im Rahmen des Qualitätsmanagements werden Arbeits- und Verfahrensanweisungen hinterlegt, sodass der Ablauf einer delegierbaren Leistung nachvollziehbar ist.
\end{abstract}

Wenn jeder „nach Vorschrift“, „nach Leitlinie“ oder „nach Ausbildungsstandard" arbeitet, sollte alles rund laufen. Leider ist das nicht so selbstverständlich. Mithin kommt es immer wieder zu Zwischenfällen und Schädigungen des Patienten. In einem solchen Fall trägt allerdings der Kläger in der Regel die Beweislast, d. h. der klagende Patient muss sowohl die schuldhafte Sorgfaltspflichtverletzung - sprich den Behandlungsfehler - als auch den Ursachenzusammenhang zwischen Fehler und Schaden beweisen.

\section{Beweiserleichterungen im Arzthaftungsprozess}

Kritisch für den beklagten Arzt oder die beklagte Gesundheitseinrichtung wird es jedoch immer dann, wenn Beweislasterleichterungen zugunsten des klagenden Patienten oder der klagenden Krankenkasse greifen. Zu den Beweiserleichterungen gemäß §630 h BGB zählen Rechtsgutverletzungen, die sich im voll beherrschbaren Herrschafts- und Organisationsbereich verwirklicht haben, Aufklärungsversäumnisse, Dokumentationsmängel, Einsatz nicht hinreichend qualifizierten Personals, grobe Behandlungsfehler sowie grobe Befunderhebungsfehler.

\section{$\S 630$ h Abs. 4 BGB - Beweislast bei Haftungs- und Aufklärungsfehler}

- (1) Ein Fehler des Behandelnden wird vermutet, wenn sich ein allgemeines Behandlungsrisiko verwirklicht hat, das für den Behandelnden voll beherrschbar war und das zur Verletzung des Lebens, des Körpers oder der Gesundheit des Patienten geführt hat.

\footnotetext{
* Ab Januar 2023 wird das MTAG durch das MTBG ersetzt: „Gesetz über die Berufe in der medizinischen Technologie“.
} 
- (2) Der Behandelnde hat zu beweisen, dass er eine Einwilligung gemäß §630 d eingeholt und entsprechend den Anforderungen des §630e aufgeklärt hat. Genügt die Aufklärung nicht den Anforderungen des $\S 630$ e, kann der Behandelnde sich darauf berufen, dass der Patient auch im Fall einer ordnungsgemäßen Aufklärung in die Maßnahme eingewilligt hätte.

- (3) Hat der Behandelnde eine medizinisch gebotene wesentliche Maßnahme und ihr Ergebnis entgegen $\S 630$ f Abs. 1 oder Abs. 2 nicht in der Patientenakte aufgezeichnet oder hat er die Patientenakte entgegen $\S 630 f$ Abs. 3 nicht aufbewahrt, wird vermutet, dass er diese Maßnahme nicht getroffen hat.

- (4) War ein Behandelnder für die von ihm vorgenommene Behandlung nicht befähigt, wird vermutet, dass die mangelnde Befähigung für den Eintritt der Verletzung des Lebens, des Körpers oder der Gesundheit ursächlich war.

- (5) Liegt ein grober Behandlungsfehler vor und ist dieser grundsätzlich geeignet, eine Verletzung des Lebens, des Körpers oder der Gesundheit der tatsächlich eingetretenen Art herbeizuführen, wird vermutet, dass der Behandlungsfehler für diese Verletzung ursächlich war. Dies gilt auch dann, wenn es der Behandelnde unterlassen hat, einen medizinisch gebotenen Befund rechtzeitig zu erheben oder zu sichern, soweit der Befund mit hinreichender Wahrscheinlichkeit ein Ergebnis erbracht hätte, das Anlass zu weiteren Maßnahmen gegeben hätte, und wenn das Unterlassen solcher Maßnahmen grob fehlerhaft gewesen wäre.

\section{Cave}

Eine Delegation ärztlicher Aufgaben auf nicht hinreichend qualifiziertes Personal stellt hierbei eine große Haftungsfalle für den Praxisbetreiber dar. Denn eine solch fehlerhafte Delegation führt dazu, dass sie als Sorgfaltspflichtverletzung eingestuft wird.

Mithin muss der klagende Patient nicht mehr das tatsächliche Fehlverhalten der handelnden Personen beweisen, sondern es reicht der Beweis aus, dass die handelnde Person, z. B. die MFA, nicht hinreichend qualifiziert war (vgl. hierzu §630a Abs. 2 BGB).

\section{$\S 630 a$ Abs. 2 BGB - Vertragstypische Pflichten beim Behandlungsvertrag (Auszug)}

- (2) Die Behandlung hat nach den zum Zeitpunkt der Behandlung bestehenden, allgemein anerkannten fachlichen Standards zu erfolgen, soweit nicht etwas anderes vereinbart ist.

Dass der Einsatz nicht hinreichend qualifizierten Personals als Sorgfaltspflichtverletzung gewertet wird, ergibt sich aus der Tatsache, dass der in §630a Abs. 2 BGB erwähnte „Standard“ auch den Anspruch des Patienten umfasst, von hinreichend qualifiziertem Personal behandelt zu werden.

Bei einer fehlerhaften Delegation kommt es hinsichtlich des Kausalitätsbeweises auch noch zu einer Beweislastumkehr zulasten der beklagten Arztpraxis oder des beklagten Arztes. Dies bedeutet, dass nunmehr der beklagte Arzt oder die beklage Arztpraxis die Beweislast dafür trägt, dass die Rechtsgutverletzung auch beim Einsatz von entsprechend qualifiziertem Personal mit an Sicherheit grenzender Wahrscheinlichkeit ebenfalls eingetreten wäre. Aufgrund des im Kausalitätsbeweis innewohnenden „Alles-oder-Nichts“-Prinzips - „Mit an Sicherheit grenzender Wahrscheinlichkeit" - wird es der beklagten Arztpraxis oder dem beklagten Arzt nur in den seltensten Fällen gelingen, einen solchen „Entlastungsbeweis“ zu führen (vgl. hierzu § 630 h Abs. 4 BGB),

\section{$\S 630$ h Abs. 4 BGB - Beweislast bei Haftungs- und Aufklärungsfehler (Auszug)}

- (4) War ein Behandelnder für die von ihm vorgenommene Behandlung nicht befähigt, wird vermutet, dass die mangelnde Befähigung für den Eintritt der Verletzung des Lebens, des Körpers oder der Gesundheit ursächlich war.

Aufgrund des Vorgesagten und der prozessualen Brisanz ist bei der Delegation von Aufgaben auf medizinisches Assistenzpersonal größte Sorgfalt geboten!

Eine Arztpraxis ohne „Arzthelferin“ oder medizinische Fachangestellte (MFA) ist kaum vorstellbar. Die heute möglichen Qualifikationen der MFAs tragen der Situation Rechnung, dass viele MFAs willens und in der Lage sind, mehr Verantwortung und delegierte Aufgaben sachadäquat zu übernehmen ( $\triangleright$ Abb. 1).

In den Hausarztpraxen hat es sich inzwischen weitestgehend etabliert, dass VERAHs (Versorgungsassistentinnen in der Hausarztpraxis) dem Arzt Routine-Hausbesuche abnehmen oder die komplette Betreuung der Wundversorgung in Altenheimen oder in der Häuslichkeit vornehmen. In den phlebologischen Praxen wurde durch die Einführung eines Kurses zur Phlebologieassistentin ein Betätigungsfeld für die MFAs eröffnet, das in diesem speziellen medizinischen Bereich deutlich mehr Verantwortung auf das Assistenzpersonal überträgt.

Wichtig in diesem Zusammenhang ist jedoch, dass eine VERAH oder eine Phlebologieassistentin immer als „Delegierte“ des Arztes unterwegs ist. Sie handelt auf Anordnung und unter der Verantwortung des sie anweisenden Arztes. Hierzu ein einführendes Fallbeispiel. 


\section{FALLBEISPIEL}

\section{Fall 1}

Die Mitarbeiterinnen einer Arztpraxis beschließen Mittwoch nach Abschluss der Sprechstunde, bei den Kolleginnen die Hepatitis-B-Impfung aufzufrischen. Die Praxisleitung (Ärztin) war bereits nach Hause gefahren.

Kollegin A wird geimpft und entwickelt wenige Minuten danach schwere Luftnot mit Zuschwellen der oberen Atemwege. Sie ist vorübergehend nicht ansprechbar, atmet schwer, allerdings selbstständig. Kollegin B ruft sofort die Praxisleitung an, Kollegin C legt parallel dazu bereits einen intravenösen Zugang und verabreicht hierüber eine $\mathrm{NaCl} 0,9 \%$-Infusionslösung. Kollegin D holt den Notfallkoffer. Unter telefonischer Anleitung der Praxisleitung wird der AmbuBeutel bereitgelegt und Dexamethason unter dem Verdacht einer akuten allergischen Reaktion intravenös verabreicht. Die Ärztin trifft ca. 10 Minuten nach dem Vorfall in der Praxis ein. Die betroffene MFA atmet weiterhin selbstständig, ferner wird sie langsam ansprechbar, Puls- und Blutdruck sind stabil. Ein Rettungswagen wird gerufen, die Patientin wird sodann ins nächstgelegene Klinikum gebracht, wo sie mit V.a. Anaphylaxie noch 2 Tage stationär behandelt wird.

\section{Fragen zum Fallbeispiel}

\section{Ist eine Hepatitis-B-Impfung delegierbar?}

Die Impfung als solche ist eine leicht durchzuführende Tätigkeit. Zur Ausbildung medizinischer Fachangestellte gehört die Verimpfung von entsprechenden Substanzen. Die Praxisleitung muss sich jedoch davon überzeugen, ob die angewiesene Person die Maßnahmen zur Durchführung der Aufgabe beherrscht. Selbstverständlich sollte dieses Befähigungsprofil dokumentiert werden. Dies war in dem obigen Fallbeispiel geschehen.

\section{War die impfende Person befugt?}

Die Praxisleitung hatte den MFAs die Fähigkeit zu impfen zugesprochen. Die Praxisleitung hat sich von der formellen und materiellen Qualifikation der Angewiesenen überzeugt. Eine Remonstration durch die Angewiesenen lag nicht vor.

\section{Wie hoch ist die Komplikationsdichte?}

Bei Impfungen sind unmittelbar auftretende Komplikationen äußerst selten, sodass sie bedenkenlos delegiert werden können. Es ist allgemein anerkannte Praxis, dass das Impfen auf eine MFA delegiert werden kann. Nach Anweisung der Praxisleitung müssen geimpfte Patienten

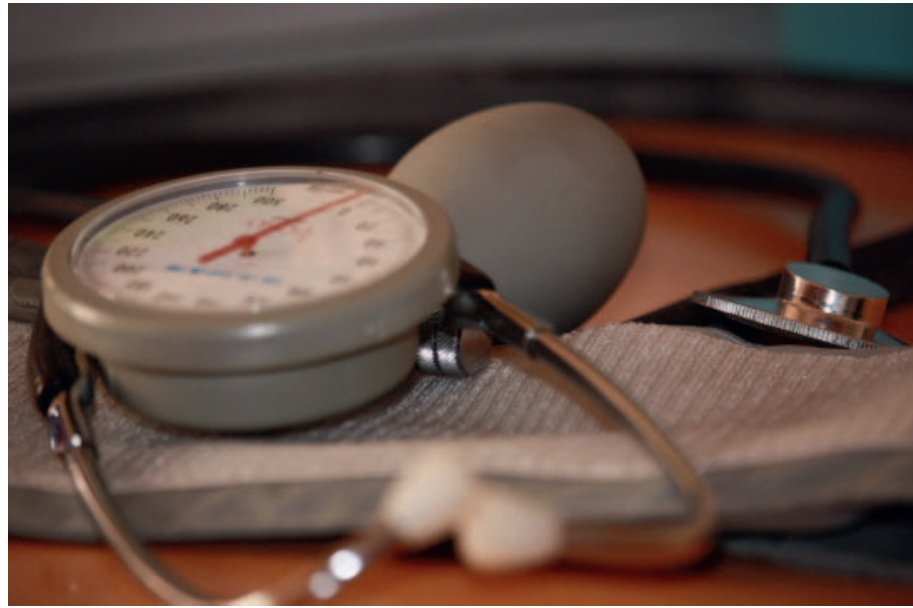

- Abb. 1 Die Blutdruckmessung ist eine typische delegierbare Leistung, ein Grundbestandteil des Handelns einer MFA. Quelle: Arrien GmbH.

30 min in der Praxis verweilen, bevor sie wieder nach Hause dürfen. Dies war in einer Arbeitsanweisung im Rahmen des QM festgelegt. Verzögert auftretende Komplikationen von Impfungen wie Fieber, Schmerz etc. sind im Rahmen der Aufklärung erklärt worden und werden in der Häuslichkeit behandelt.

Wie schnell muss im Fall einer Komplikation der Arzt eingreifen können?

Bei einem anaphylaktischen Schock muss der Arzt sofort eingreifen, d. h. es darf nur geimpft werden, wenn der Arzt im Hause ist (so auch die Arbeitsanweisung). Auch Mitarbeiter untereinander werden zu Patienten, wenn sie die Empfänger der Impfung sind, daher gilt auch für sie: Bei einer Impfung muss der Arzt anwesend sein, um bei Komplikationen sofort eingreifen zu können.

\section{Erfreulicherweise ist durch die Abwesenheit des Arztes kein Schaden entstanden.}

Weil die anwesenden Mitarbeiter unmittelbar und beherzt korrekt gehandelt und nach Rücksprache sowie telefonischer Anweisung durch die Ärztin die notwendigen Maßnahmen ergriffen haben, ist kein Schaden für die Patientin entstanden.

\section{Juristische Konsequenz bezogen auf das Fallbeispiel}

Wäre es in diesem Fall zu einem schädigenden Ereignis gekommen, wäre die Frage der Verantwortung zu klären. Da im Praxis-QM klar definiert ist, dass die Impfung nur stattfinden darf, wenn der Arzt im Hause verweilt, wäre die MFA in der vorliegenden Fallkonstellation alleinig verantwortlich gewesen, da sie gegen die ausdrücklich im Praxis-QM hinterlegte Anweisung der Ärztin verstoßen hat. 


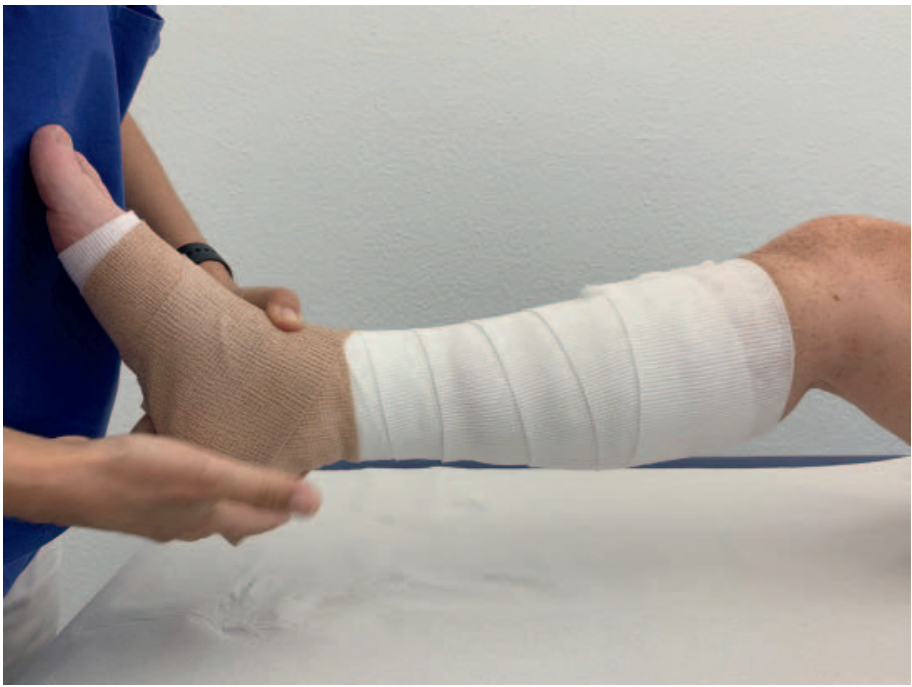

- Abb.2 Das Bandagieren der Wade bei Ödem ist eine delegierbare Leistung. Abgesehen von den während der Ausbildung vermittelten Fertigkeiten muss die ausführende Person aber Kenntnisse über das verwendete Material sowie dessen Handhabung und über die Wickeltechniken bei verschiedenen Sorten von Ödem (z. B. Lymphödem ab Zehenspitzen, Wadenödem wie hier ab Vorfuß) besitzen. Diese Kenntnisse sollten ständig aktualisiert werden und in Weiterbildungen wie auch praktisch geübt werden. Quelle: Arrien GmbH.

\section{FAZIT}

\section{Take Home Message}

Kommen solche Verstöße gegen schriftliche Anweisungen des Arztes allerdings öfter vor und erlangt der Arzt hiervon Kenntnis, ist es zwingend geboten, diesem Verhalten Einhalt zu gebieten. Im Rahmen der ärztlichen Aufsichtspflicht und im Rahmen des Direktionsrechts des Arbeitgebers sind entsprechende Maßnahmen zu ergreifen, um anweisungswidriges Verhalten zu unterbinden. Neben Ermahnungen können - abhängig von der Fallkonstellationen auch arbeitsrechtliche Abmahnungen als Sanktionen herangezogen werden.

Beispiel einer schriftlichen Anweisung, welche im Praxis-QM hinterlegt werden sollte

Die medizinischen Fachangestellten dürfen folgende Aufgaben im Rahmen der Delegation nach ausdrücklicher Anweisung durch den behandelnden Arzt eigenständig wahrnehmen:

- Blutdruckmessen,

- Verbandswechsel,

- Blutentnahme,

- subkutane Injektionen folgender Wirkstoffe: ... (Wirkstoffe aufführen) und

- Ausführen weiterer Aufgaben, die einer Delegation zugeführt werden können.

\section{Aufgabenzuordnung}

Jährlich werden rund 11000 Behandlungen, bei denen Behandlungsfehler vermutet werden, durch die Gutachterkommissionen und Schlichtungsstellen bei den Ärztekammern im Hinblick auf die Arzthaftung bewertet [1] Gegenstand der Auseinandersetzung ist immer wieder der Vorwurf der fehlerhaften Strukturierung der Arbeitsabläufe. Daher ist die klare Abgrenzung von Zuständigkeitsbereichen in einem arbeitsteiligen ärztlich/pflegerischen Behandlungsprozess von eminenter Bedeutung. Dies ist maßgeblicher Bestandteil des gesetzlich vorgeschriebenen Praxis-Qualitätsmanagements (QM). Die Organisation und die fachlich-strukturelle Gliederung der Dienste müssen sicherstellen, dass die ärztliche Gesamtverantwortung von den Aufgaben des nichtärztlichen Personals deutlich abgegrenzt wird.

In der Vergangenheit orientierte sich die Abgrenzung der Kompetenzbereiche an Grundsätzen der Rechtsprechung, da der bundesdeutsche Gesetzgeber keine eindeutige Abgrenzung der Verantwortungssphären zwischen Ärzten und anderen Gesundheitsberufen vorgesehen hatte. Wenngleich durch das Pflege-Weiterentwicklungsgesetz seit dem 1.07.2008 Pflegefachkräfte in speziellen Modellprojekten gemäß §63 SGB V als eigenständige Leistungserbringer im Gesundheitswesen anerkannt werden können, die selbstständig zur Ausübung der Heilkunde berechtigt sind (vgl. hierzu §63b u. §63c SGB V), hat diese Entwicklung keine unmittelbare Auswirkung auf das herkömmliche Verantwortungsgeflecht zwischen Ärzten und Pflegefachkräften.

\section{Delegation auf praxisexterne Dienstleister}

Gemäß dem Vorgesagten entsteht bei der Delegation von Aufgaben auf praxisexterne Dienstleister ein besonderes Verantwortungsgeflecht. Bezüglich der Übertragung von Aufgaben auf Dritte sind z. B. folgende Handlungen betroffen:

- das Verabreichen von Medikamenten durch die häusliche Krankenpflege,

- das Vornehmen von Verbandswechseln und

- das Bandagieren von Beinen etc. [2, 3].

Zunächst ist festzustellen, dass die Handlung auf Anweisung des Arztes erfolgt, der grundsätzlich im Rahmen seiner Anordnungsverantwortung die subjektive Fähigkeit des Angewiesenen zu überprüfen hat. Soweit die Delegation der Aufgaben sich im Rahmen des Ausbildungsprofils des Angewiesenen bewegt, kann der delegierende Arzt davon ausgehen, dass die angewiesene Maßnahme, z. B. das Bandagieren des Beines, von der angewiesenen Person sachgerecht durchgeführt wird ( Abb. 2). 
Es ist nicht möglich, dass der Arzt sich von jedem Mitarbeiter des Pflegedienstes zeigen lässt, dass er entsprechend der Ausbildung die Maßnahmen auch tatsächlich technisch korrekt beherrscht bzw. auch das Material kennt, welches der Arzt zum Bandagieren verordnet hat. Der Arzt haftet daher bei der Delegation von Maßnahmen an externe Dienstleister im Rahmen seiner Durchführungsverantwortung nur für die Verordnung des korrekten Materials und die korrekte Therapieanordnung - z. B. darf bei akutem Erysipel keine Bandagierung verordnet werden. Sind sowohl die Indikation und das verordnete Material korrekt als auch die Anordnung auf der Verordnung ausreichend klar formuliert, trifft im Schadensfall die haftungsrechtliche Verantwortung den externen Dienstleister, beispielsweise beim Auftreten eines Druckulkus, weil die Bandage zu festgezogen wurde.

\section{Delegation innerhalb der Arztpraxis}

Abseits der eigenverantwortlichen Ausübung von heilkundlichen Aufgaben durch Pflegefachkräfte (Allokation) gilt in einem zivilgerichtlichen Haftungsprozess wegen eines Behandlungsfehlers, der im Zuge der Ausführung einer delegierten Maßnahme verursacht worden ist, nach wie vor das Anordnungsmonopol der Ärzte. Der delegierende Arzt muss also genau festlegen, welche Aufgaben delegierbar sind und ob diese tatsächlich innerhalb der konkreten Praxissituation übertragen werden können und wer als „Delegat“ (sprich: Person, die die Leistung ausführt) ausreichend qualifiziert ist. Das bedeutet:

Merke

Bestimmte Aufgaben dürfen keineswegs und andere nur unter gewissen Voraussetzungen an nichtärztliches Personal übertragen werden.

\section{Vertikale Arbeitsteilung}

Betroffen von dem Aufgabentransfer sind zahlreiche Aufgabenfelder, da eine Vielzahl von medizinischen Behandlungsmaßnahmen (z. B. subkutane Injektionen, das Wechseln eines Wundverbandes oder die Gabe von Medikamenten) nicht direkt von Ärzten, sondern im Rahmen der vertikalen Arbeitsteilung durch nichtärztliches Personal erbracht wird. Diese sogenannte vertikale Arbeitsteilung ist durch ein Unter- und Überordnungsverhältnis gekennzeichnet, welches auf der jeweiligen Fachqualifikation und/oder Weisungsgebundenheit des angewiesenen Personals gründet [4]. Horizontale Arbeitsteilung hingegen meint die Aufteilung von Aufgaben innerhalb einer Berufsgruppe.

Die vertikale Arbeitsteilung beinhaltet die komplette Praxisorganisation beginnend mit der Terminvergabe am Telefon, der Annahme des Patienten in der Praxis, der Eingabe der Daten mit Anamneseerhebung bis hin zur Ausführung der technischen Untersuchungen. Ferner werden die Durchführung von Verbandswechsel, Injektionen oder komplexe Vorgänge wie das Führen von eigenen Sprechstunden (Ernährungsberatung, Betreuung von chronischen Wunden) vom medizinischen Assistenzpersonal übernommen.

\section{Merke}

Das Stellen einer Diagnose ist dabei eine Aufgabe, die niemals delegiert werden darf, auch wenn alle Schritte, die zur Diagnose führen, von MFAs durchgeführt werden können und sie mit der entsprechenden Erfahrung die Diagnose am Ende auch kennen.

Zum besseren Verständnis ein Beispiel:

\section{Verantwortungsbereich von Phlebologieassis- tenten bei der Notfallvorstellung eines Patienten zum Ausschluss einer Thrombose}

Phlebologieassistent*innen sind qualifiziert

- zur Umfangsmessung von Beinen,

- zur Palpation,

- zur Inspektion,

- zur Erhebung der einschlägigen Anamnese (WellsScore) sowie

- zur Durchführung eines D-Dimers bei positivem WellsScore.

In einigen Fällen sind sie sogar ausgebildet, Sonografiebefunde zu erheben und zu dokumentieren.

Die Diagnose „Thrombose“, die dann möglicherweise schon offensichtlich ist und sich dem Delegaten auch erschließt, darf aber nur der Arzt in der Gesamtschau der erhobenen Befunde und unter persönlichem Betätigen des Schallkopfes auf der Thrombose selbst stellen.

Tatsächlich wird das in anderen Ländern anders gehandhabt: Dort gibt es sogenannte „Technicians“, die die komplette technische Diagnostik inklusive des Ultraschalls durchführen und die Diagnosen selbst stellen. In Deutschland ist dies jedoch derzeit so nicht vorgesehen.

Auch die auf der Diagnose fußende Therapie ist dem alleinigen Verantwortungsbereich des Arztes zuzuordnen. Nur die der Therapie zugrunde liegenden Behandlungsmaßnahmen können unter den unten aufgezeigten Voraussetzungen einer Delegation zugeführt werden.

Wenngleich die Effektivität und der ökonomische Nutzen einer Aufgabenteilung generell unbestritten sind, werden zugleich die Reichweite bzw. die Grenzen einer zulässigen Delegation seit Jahrzehnten unter Gesundheitsrechtlern diskutiert. Nur wenige Rechtsnormen schreiben ausdrücklich das Tätigwerden des Arztes vor. Das ist abgesehen von der Diagnosestellung z. B. der Fall bei der An- 
wendung von Blutprodukten, geregelt in z. B. §13 Transfusionsgesetz (TFG) [5]:

\section{$\S 13$ TFG - Anforderungen an die Durchführung (Auszug)}

- (2) Die ärztlichen Personen, die eigenverantwortlich Blutprodukte anwenden, müssen ausreichende Erfahrung in dieser Tätigkeit besitzen.

Hier gilt als „gesetzt“ der Ausbildungsstandard des jeweiligen Berufes (insbesondere medizinische Fachangestellte für die Arztpraxis). Aus dem Ausbildungsziel des neuen Pflegeberufsgesetzes (PflBG) leitet sich die Befähigung der Pflegefachfrau oder des Pflegefachmanns zur eigenständigen Durchführung ärztlich angeordneter Maßnahmen ab (vgl. § 5 Abs. 3 Nr. 2 PflBG) - eine Beschreibung, welche Maßnahmen darunter fallen, findet sich allerdings weder an dieser noch an anderer Stelle des Gesetzes. Mögliche Anhaltspunkte zur Definition der Kompetenzbereiche können deshalb nur der juristischen Literatur, den Stellungnahmen der Berufs- und Fachverbände und nicht zuletzt der Rechtsprechung entnommen werden.

\section{$\S 5$ Abs. 3 Nr. 2 PflBG - Ausbildungsziel (Auszug)}

- (3) Die Ausbildung soll insbesondere dazu befähigen, - 1. [...]

- 2. ärztlich angeordnete Maßnahmen eigenständig durchzuführen, insbesondere Maßnahmen der medizinischen Diagnostik, Therapie oder Rehabilitation [6].

\section{FAZIT}

\section{Take Home Message}

Aus Sicht der Arztpraxis ist es häufig prozessentscheidend, dass das eingesetzte Personal in einem Haftungsprozess als hinreichend befähigt eingestuft wird. Insbesondere ist es erforderlich, die materielle Qualifikation des zum Einsatz gebrachten Personals gerichtsfest darstellen zu können. Die tatsächlichen Fähigkeiten des eingesetzten Personals sollten insoweit durch strukturierte Fort- und Weiterbildungspläne und entsprechende Befähigungsnachweise transparent und nachvollziehbar sein.

Eine entsprechende Dokumentation ist zwingend erforderlich. Nur so kann der Gefahr begegnet werden, dass vonseiten des Gerichts die materielle Qualifikation verneint wird. Impulse für die Beantwortung der Fragestellung nach der Reichweite der Delegation von Maßnahmen an Assistenzpersonal sowie angelernte Kräfte folgen aus einer Entscheidung des SG Speyer [7].
Aus der haftungsrechtlichen Diskussion haben sich folgende Kriterien, die bei einer Übertragung von Aufgaben berücksichtigt werden müssen, herausgebildet:

Die objektive Gefährlichkeit der angewiesenen Maßnahme umfasst z. B. den Risikograd des Eingriffs, die Häufigkeit der möglicherweise auftretenden Komplikationen sowie die Gefährdungsnähe der angewandten Technik. Je höher das Risiko und je häufiger die Möglichkeit der Komplikation, desto höher ist die Gefährlichkeit einzuschätzen. Diese Maßnahmen sind dann eher nicht delegierbar.

Die subjektiven Fähigkeiten des Angewiesenen bezieht sich auf die Fähigkeit des Angewiesenen sowohl in materieller als auch formeller Hinsicht.

- Die formelle Qualifikation umfasst die Fähigkeiten, welche durch die Ausbildung und die Weiterbildungen erworben werden. Mithin stellen die Ausbildungs- und Prüfungsordnungen der einzelnen Fachberufe eine wesentliche Erkenntnisquelle dafür dar, was überhaupt übertragen werden kann.

- Selbstverständlich muss der Angewiesene das Erlernte auch fehlerfrei in der Praxis zur Anwendung bringen können. Hierunter ist die materielle Qualifikation zu verstehen. Daher muss der delegierende Praxisinhaber sich immer davon überzeugen, dass der Angewiesene die übertragende Aufgabe auch tatsächlich beherrscht. Dieser Vorgang sollte dann im Rahmen des QM oder der Personalakte hinterlegt werden.

Bei der Verabreichung einer Impfung gehören zur Beherrschung der Aufgabe

- die ordnungsgemäße Beherrschung der Impftechnik,

- Informationen über Wirkungen und Nebenwirkungen des verabreichten Wirkstoffs sowie

- die Fähigkeit der ausführenden Fachkraft, die Reaktion des Patienten richtig deuten zu können.

\section{Delegationsmodell am Beispiel einer Injektion}

Die zu prüfenden Delegationsvoraussetzungen sollen am Beispiel der Delegation der Verabreichung eines Wirkstoffs durch Injektion dargestellt werden: Bei der Prüfung der objektiven Gefährlichkeit ist im Rahmen der Komplikationsdichte und Gefährdungsnähe auch der Zustand des Patienten zu berücksichtigen. Ferner sollten Negativkataloge bezüglich der zu verabreichenden Wirkstoffe praxisbezogen festgelegt werden.

Im Rahmen der subjektiven Fähigkeit des Angewiesenen muss neben der formellen Qualifikation auch die materielle Qualifikation zwingend beachtet werden. Neben der Beherrschung der Injektionstechnik müssen grobe Kenntnisse über die Wirkungen und Nebenwirkungen des verabreichten Wirkstoffs beim Angewiesenen vorliegen. Sowohl die Krankenbeobachtung als auch Erste-Hilfe-Maßnahmen müssen vom Angewiesenen beherrscht werden. Die mate- 
riellen Fähigkeiten können durch einen Befähigungsnachweis schriftlich hinterlegt und der Dokumentation zugeführt werden.

\section{Bedeutung für die Praxis}

Eine Delegation ärztlicher Aufgaben verbietet sich immer dann, wenn mit der Maßnahme eine derart hohe Komplikationsdichte verbunden ist, dass diese nur durch ärztliches Fachwissen und -können zu beherrschen wäre. Umgekehrt gilt jedoch: Je geringer die Komplikationsdichte und Gefährdungsnähe, desto eher kann die Maßnahme auch delegiert werden [8].

Wird die Zulässigkeit der Delegation einer Maßnahme nun grundsätzlich bejaht und ist der richtige Adressat ausgewählt, stehen den positiven Effekten des arbeitsteiligen Vorgehens die bekannten Gefahren wie beispielsweise Koordinations- und Kommunikationsfehler, Organisations- und Kontrollfehler gegenüber. In diesem Zusammenhang ist fraglich, welche Sorgfaltspflichten für den Delegierenden erwachsen und wie diese ausgefüllt werden müssen.

\section{Anordnungsverantwortung}

Steht die Delegationsfähigkeit einer Maßnahme außer Frage und ist auch der richtige, also der zur Übernahme entsprechend qualifizierte Delegationsadressat ausgewählt worden, so ist der Arzt für eine ordnungsgemäße Anordnung der Maßnahme verantwortlich. Anordnungsverantwortung, Instruktions- und Überwachungspflicht sind dabei Bestandteil der ärztlichen Gesamtverantwortung, die trotz arbeitsteiligem Vorgehen beim Arzt verbleibt.

\section{FALLBEISPIEL}

Fall 2

Einer Entscheidung des OLG Dresden (s. RDG 2008, S. 240 ff. [9]) liegt die Behauptung der Delegation an nicht hinreichend qualifiziertes Personal zugrunde. Eine Patientin hat die Ärzte einer radiologischen Gemeinschaftspraxis als Gesamtschuldner auf Schmerzensgeld und Feststellung der Ersatzpflicht für die Folgen einer Technetium-Lösung in die Vene ihrer rechten Ellenbeuge in Anspruch genommen. Die Injektion wurde von der leitenden medizinischtechnischen Radiologieassistentin (MTRA) vorgenommen. Die Patientin behauptete unter anderem, dass diese nicht über das erforderliche Qualifikationsprofil verfüge und derartige Injektionen generell dem Arzt vorbehalten seien.

Das Landgericht Dresden hat die Klage abgewiesen (Az.: 6 O 3070/03). Auch die Berufung der Patientin war nicht erfolgreich. Das Oberlandesgericht Dres- den urteilte, dass die Delegation der Injektion auf die MTA nicht behandlungsfehlerhaft gewesen sei. Es handelte sich einerseits um eine erfahrene und fachgerecht ausgebildete Kraft (materielle und technische Qualifikation), und andererseits war sie zur Verabreichung auch befugt (Arbeitsanweisung).

Wie alle Arbeiten einer ärztlichen Praxis werden zwar derartige Injektionen dem ärztlichen Verantwortungsbereich zugerechnet. Es handelt sich hierbei aber nicht um eine Tätigkeit, die aufgrund ihrer Schwierigkeit, Gefährlichkeit oder Unvorhersehbarkeit zwingend von einem Arzt erbracht werden muss, und daher ist die Aufgabe delegierbar. Die Risiken der Technetium-Injektion wurden mit denen einer Blutentnahme verglichen, die ebenfalls regelmäßig auf nichtärztliche Fachkräfte übertragen werden kann ( $\triangleright$ Abb. 3). Hinzu trat, dass im Rahmen der Berufsausbildung zur MTA die für die Verabreichung einer Injektion erforderlichen medizinischen Grundlagenkenntnisse vermittelt werden (§3 Nr. 2 MTAG). Da die behaupteten Irritationen (Verletzung der A. brachialis mit Ausbildung eines Hämatoms in der Ellenbogenbeuge) auch bei größter Sorgfalt nicht immer vermeidbar sind, wurde die Berufung zurückgewiesen [9].

Aus dem vorbezeichneten Fallbeispiel 2 lassen sich folgende Delegationsgrundsätze herausarbeiten (die nachfolgende Checkliste kann für delegierbare Leistungen herangezogen werden; dabei ist es wichtig, dass im Rahmen der Beurteilung der objektiven Gefährlichkeit jeweils eine Begründung angeführt werden sollte, wieso die Aufgabe nicht dem Kernbereich ärztlichen Handelns zuzurechnen ist):

\section{Checkliste}

- Übertragbarkeit der Aufgabe (objektive Gefährlichkeit)

- Gehört die zu übertragende Aufgabe zum Kernbereich medizinischer (und daher nicht delegierbarer) Behandlung? Dies ist abhängig von:

- hoher Schwierigkeit,

- hohem Risiko und

- Unvorhersehbarkeit etwaiger Reaktionen.

- Sind die Schwierigkeit und das Risiko nicht hoch und die etwaigen Reaktionen vorhersehbar, ist die Aufgabe grundsätzlich übertragbar. Im vorliegenden Fall kann die bei sachgerechter Durchführung sehr selten auftretende Verletzung eines benachbarten Gefäßes in Kauf genommen werden, zumal die Folgen (Hämatom) gering sind.

- Beherrschung durch Delegaten (subjektive Fähigkeit)

- Formelle Qualifikation (Ausbildungsnachweise, die auf eine bundes- oder landesrechtliche Ausbildungsregelung fußen): Die MTA war durch ihre Ausbildung zu der Anlage eines Zugangs in der 


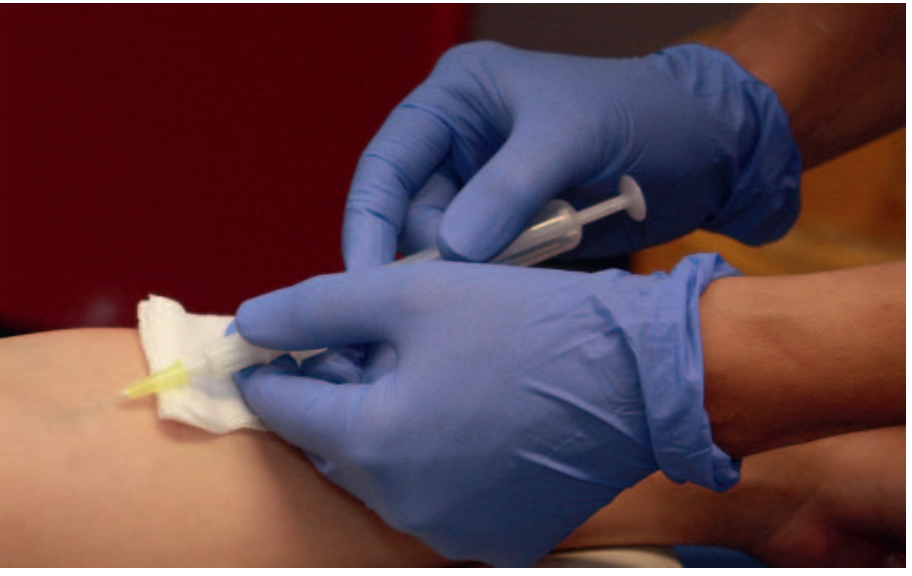

- Abb. 3 Die Blutentnahme ist eine für Arzthelferinnen übliche Aufgabe, die zu den klassischen delegierbaren Leistungen gehört. Allerdings ist der Schwierigkeitsgrad höher als der einer Blutdruckmessung. Quelle: Arrien $\mathrm{GmbH}$.

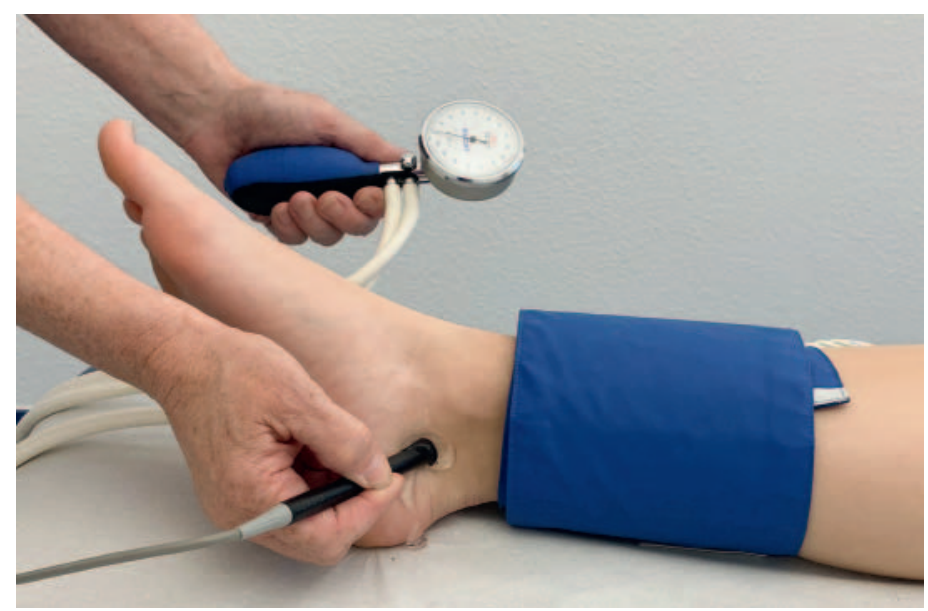

- Abb. 4 Messung des Knöchel-Arm-Index: Der Blutdruck am Knöchel und am Fußrücken wird mit einer Doppler-Stiftsonde (hier: korrekte Haltung der Stiftsonde nicht senkrecht zur Haut, sondern in Richtung der Arterie) und einem Blutdruckmessgerät abgeleitet. Quelle: Arrien GmbH.

Armbeuge und zur Infusion eines Kontrastmittels auf Anweisung des verantwortlichen Arztes qualifiziert.

- Materielle Qualifikation (persönliche Überzeugung der Fähigkeiten des Mitarbeiters): Der verantwortliche Arzt hat sich im Rahmen der Probezeit der MTA sowie danach im täglichen gemeinsamen Arbeiten wiederholt davon überzeugen können, dass die MTA die Anlage der Infusion beherrscht. Im besten Fall ist dies auch im QM oder in der Personalakte hinterlegt (Beispiel in $>$ Abb. 4).

- Anordnung des Arztes (in der Regel schriftlich) oder in Abläufen festgelegt - im vorliegenden Fall gibt es eine Arbeitsanweisung, dass bei Magnetresonanztomogra- fie mit Anwendung einer Infusion die MTA diese Infusion anlegt; in anderen Fällen z. B. auch die generelle Untersuchung z. B. von Puls und Blutdruck bei neuen Patienten.

- Remonstrationsrecht: Dies regelt das Recht des Delegaten (sprich der Person, die die Aufgabe übertragen bekommt), diese abzulehnen und dazu auch ausreichend Gelegenheit zu bekommen ( $\rightarrow$ allgemeines Remonstrationsrecht des Delegaten immer beachten!).

- Ärztliche Dichte (Wie schnell kann der anordnende Arzt im Komplikationsfall einschreiten?) Für das erste Fallbeispiel (Impfung), bei der die Komplikation sofort eintreten kann, muss der Arzt im Hause sein. Für das Beispiel mit der Anlage der Infusion bei Magnetresonanztomografie ist der Arzt ohnehin im Haus, da sonst die Untersuchung nicht durchgeführt würde. Die Komplikation trat ohnehin zeitverzögert auf (Hämatombildung) und hätte im ersten Moment nicht beobachtet oder vermieden werden können durch die Anwesenheit eines Arztes.

\section{Beispiel: Messung des Knöchel-Arm-Index}

Die Messung des Knöchel-Arm-Index zur Diagnostik bei Verdacht auf arterielle Verschlusskrankheit ist eine delegierbare Leistung. Dabei wird der Blutdruck am Knöchel und am Fußrücken mit einer Doppler-Stiftsonde und einem Blutdruckmessgerät abgeleitet ( $\bullet$ Abb. 4). Allein die korrekte Haltung der Stiftsonde (nicht senkrecht zur Haut, sondern in Richtung der Arterie, wie hier im Bild zu sehen) ist Übungssache und erfordert umfangreiche spezifische Kenntnisse. Das Ergebnis ist sehr stark von der korrekten Durchführung abhängig. Die reine Ausbildung zur medizinischen Fachangestellten reicht nicht aus. Die Mitarbeiter müssen vom Praxisinhaber speziell geschult werden bzw. diese Schulung muss sichergestellt werden. Der Arzt muss sich persönlich versichern, dass die Untersuchung beherrscht wird.

\section{FAZIT}

\section{Take Home Message}

Hat die Überprüfung des Sachverständigen ergeben, dass die übertragene Aufgabe bereits wegen objektiver Gefährlichkeit nicht übertragbar ist, so ist die mit der Aufgabenwahrnehmung beauftragte MFA nicht hinreichend qualifiziert, was sich zugunsten der klagenden Partei auf der Sorgfaltspflichtverletzungs- und Kausalitätsebene beweiserleichternd auswirkt. 


\section{FALLBEISPIEL}

\section{Fall 3 - Beispiel für fehlende Qualifikation}

Die Klägerin begab sich zur Vornahme eines plastischen Eingriffs, der unter Vollnarkose durchgeführt werden sollte, in die Klinik des Beklagten. Nach erfolgtem Eingriff wurde die Klägerin zur postoperativen Betreuung auf ein Patientenzimmer verbracht. Die Versorgung zur Nacht übernahm eine (ebenfalls beklagte) Medizinstudentin im 10. Semester; sie war als einzige Nachtwache vorgesehen. Bei der Übergabe durch den Anästhesisten schrieb dieser eine Medikamentenliste, die u. a. auch den Eintrag „Infusionsrest aus OP i. v.“ enthielt. Nachdem die Klägerin sich in der Nacht mehrfach hat übergeben müssen, infundierte die Medizinstudentin den zuvor aus dem OP geholten Tropf. Die Infusionsflasche, die mit „NaCl“ beschriftet war, enthielt aber außer Kochsalzlösung auch das Medikament Propofol, womit der Anästhesist die Narkose durchgeführt hatte. Die Klägerin geriet in einen reanimationspflichtigen Zustand und verblieb - nach notärztlicher Versorgung - im Zustand des Wachkomas.

Das LG Mainz stellte später fest, dass die Medizinstudentin für die alleinige postoperative Überwachung medizinisch nicht geeignet war. Ein Medizinstudent im 10. Semester hat ganz überwiegend theoretische Kenntnisse erworben. Es fehlt ihm aber regelmäßig an der praktischen Ausbildung für den Dienst am Patienten, so die Richter. Schon mit der eigentlich geringfügigen Komplikation einer Übelkeit war die Medizinstudentin hoffnungslos überfordert.

Mithin ist der Fall zu Lasten der Gesundheitseinrichtung entschieden worden.

\section{Durchführungsverantwortung}

Das angewiesene Personal trägt bezüglich der Aufgabenerledigung immer die sogenannte Durchführungsverantwortung. Hierbei handelt es sich um die Verantwortung, die angewiesene Maßnahme sach- und fachgerecht zu erledigen. Mithin ist es aus Sicht des Ausführenden von Bedeutung, dass im Rahmen einer Eigenkontrolle festgestellt wird, ob der Ausführende aufgrund seiner formellen und materiellen Qualifikation in der Lage ist, die angewiesene Maßnahme ordnungsgemäß durchzuführen. Kommt es bei der Durchführung einer Maßnahme zu einer Schädigung des Patienten und ist diese Schädigung auf ein Fehlverhalten des Durchführenden zurückzuführen, so ist der Handelnde für den entstandenen Schaden zur Verantwortung zu ziehen [10]. In diesem Fall ist tatsächlich der Arzt von der Haftung befreit.

\section{PRAXIS}

\section{Zu beachten}

Das angewiesene Personal sollte wissen, dass es die Ausführung einer Aufgabe verweigern darf, wenn es sich z. B. nicht ausreichend auf diese Aufgabe vorbereitet fühlt oder wenn die angewiesene Person die Situation anders einschätzt als der Beauftragende und sich daher der Situation nicht gewachsen fühlt. Das bedeutet „fehlende Durchführungskompetenz“ (real oder vom Ausführenden so empfunden): Im Falle der fehlenden Durchführungskompetenz sollte jeder von seinem Recht Gebrauch machen dürfen, die Übernahme der Aufgabe zu verweigern. Mitarbeiter sollten von dem Praxisleiter bestärkt und unterstützt werden, bei fehlender eigener Kompetenz dies auch gegenüber dem Anweisenden zum Ausdruck bringen zu dürfen ohne mit negativen Konsequenzen rechnen zu müssen.

Das Recht, eine delegierte Aufgabe zu verweigern, heißt Remonstrationsrecht.

\section{Schlussfolgerung}

Delegierbare Leistungen sind keine Selbstverständlichkeit, auch wenn es sich oft so anfühlt. Arzthelferinnen oder MFAs „sind dazu da“, subkutane Injektionen zu verabreichen oder geschwollene Beine zu wickeln. Die Anordnungen zur korrekten Durchführung (Indikation, Material, Ausführung, Erläuterungen an den Patienten) müssen im Rahmen des QM der Praxis hinterlegt werden.

\section{PRAXIS}

Tipp

Ob diese Fähigkeiten von jedem Mitarbeiter in der Ausbildung erlernt und in der Praxis korrekt angewandt werden können, muss vom verantwortlichen Arzt festgestellt und nachvollziehbar dokumentiert werden. Es ist sinnvoll, ein solches Befähigungsprofil für jeden Mitarbeiter zu führen. Nur so ist man in einem Haftungsprozess gegen mögliche Ansprüche klagender Patienten gewappnet.

\section{KERNAUSSAGEN}

- Bei der Delegation ärztlicher Aufgaben auf nichtärztliches Personal muss zunächst festgestellt werden, ob die Aufgabe überhaupt delegierbar ist oder dem Kernbereich ärztlichen Handelns zugewiesen werden muss. Bei der Beurteilung der Delegierbarkeit einer Aufgabe sind die Komplikationsdichte und die Gefährdungsnähe einer 
Aufgabe einer kritischen Beurteilung durch den Anweisenden zuzuführen.

- Ist eine ärztliche Tätigkeit als delegierbar eingestuft worden, muss der Delegationsempfänger hinreichend qualifiziert sein. Es ist mithin die formelle und materielle Qualifikation zu überprüfen.

- Die formelle Qualifikation beschreibt die erlernten Fähigkeiten des Delegaten, die auf einer bundes- oder landesrechtlichen Ausbildungsregelung fußen.

- Die materielle Qualifikation beschreibt die tatsächlichen Kenntnisse und Fähigkeiten des Delegaten.

- Bei der Delegation ärztlicher Aufgaben trägt der Anweisende die Anordnungsverantwortung, welche die Auswahlverantwortung (Auswahl des richtigen Anordnungsadressaten), die Handlungsverantwortung (Auswahl der richtigen Maßnahme) und die Kontrollverantwortung (Kontrolle des Anordnungsadressaten) umfasst.

- Neben der Anordnungsverantwortung des Angewiesenen trägt das durchführende Personal die sogenannte Durchführungsverantwortung. Die Durchführungsverantwortung umschreibt die Sorgfaltsverantwortung (die Verantwortung zur sachadäquaten Ausführung der übertragenen Aufgabe) und die Übernahmeverantwortung (Selbstprüfung des Durchführenden, ob er in der Lage ist, die Maßnahme ordnungsgemäß zu erledigen).

- Sollte der Angewiesene sich nicht imstande fühlen, die angewiesene Aufgabe sachadäquat zu erfüllen, hat er das Recht zu remonstrieren. Remonstration bedeutet, dass der Angewiesene das Recht hat, die ihm übertragene Aufgabe zurückzuweisen.

\section{Wissenschaftlich verantwortlich gemäß Zertifizierungsbestimmungen}

Wissenschaftlich verantwortlich gemäß Zertifizierungsbestimmungen für diesen Beitrag ist Dr. med. Erika Mendoza, Wunstorf.

\section{Interessenkonflikt}

Erklärung zu finanziellen Interessen

Forschungsförderung erhalten: nein; Honorar/geldwerten Vorteil für Referententätigkeit erhalten: ja, von einer anderen Institution; Bezahlter Berater/interner Schulungsreferent/Gehaltsempfänger: ja, von einer anderen Institution; Patent/Geschäftsanteile/Aktien (Autor/Partner, Ehepartner, Kinder) an Firma (Sponsor der Veranstaltung): nein; Patent/Geschäftsanteile/Aktien (Autor/Partner, Ehepartner, Kinder) an Firma (Nicht-Sponsor der Veranstaltung): nein.
Erklärung zu nichtfinanziellen Interessen

Erika Mendoza: Generalsekretärin der DT. Gesellschaft für Phlebologie Vorsitzende der folgenden Gesellschaften: Wundnetz Hannover West e. V., Dt. Gesellschaft für CHIVA ge. V., Hospizverein Schaumburg Lippe e. V:

\section{Autorinnen/Autoren}

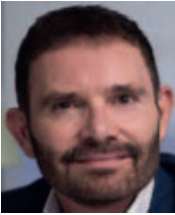

\section{Volker Großkopf}

Prof. Dr. jur., 1983-1989 Studium der Rechtswissenschaft an der Rheinischen Friedrich-Wilhelms-Universität Bonn. Rechtsanwalt und Professor für Rechtswissenschaften an der Katholischen Hochschule Nordrhein-Westfalen (KatHO NRW), Fachbereich Gesundheitswesen, Abteilung Köln. Leiter des gesundheitsrechtlichen Fortbildungsinstituts PWG-Seminare sowie Initiator des ,JuraHealth Congress“ (JHC) und des „Interdisziplinären WundCongress“ (IWC). Autor zahlreicher medizin- und pflegerechtlicher Fachartikel und Fachbücher, Herausgeber der Fachzeitschrift „Rechtsdepesche für das Gesundheitswesen“.

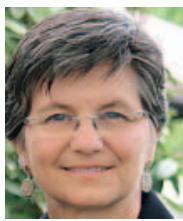

\section{Erika Mendoza}

Dr. med., Studium der Medizin in Madrid (Universidad Autónoma) bis 1990; 1994 Fachärztin für Allgemeinmedizin, Madrid, Spanien. 1995-1996 tätig als Allgemeinärztin in eigener Praxis in Trujillo, Spanien. Erlernen der DuplexSonografie zur Durchführung der CHIVAMethode in Barcelona, Paris, Ferrara. Seit 1997 tätig in eigener Praxis in Wunstorf/Hannover mit Schwerpunkt Phlebologie. 2003 Weiterbildung in Palliativmedizin an der Mildred-ScheelAkademie, Köln.

Korrespondenzadresse

Rechtsanwalt Prof. Dr. jur. Volker Großkopf

PWG-Seminare

Salierring 48

50677 Köln

Deutschland

grosskopf@rechtsdepesche.de

Literatur

[1] Bundesärztekammer Arbeitsgemeinschaft der deutschen Ärztekammern. Statistische Erhebung der Gutachterkommissionen und Schlichtungsstellen für das Statistikjahr 2019. Im Internet (Stand: 11.08.2021): https://www.bundesaerztekam mer.de/fileadmin/user_upload/downloads/pdf-Ordner/ Behandlungsfehler/Statistische_Erhebung_2019.pdf

[2] Großkopf/Schanz. Heilkundeübertragung-vor, während und nach Corona, in RDG 2020, Seite $126 \mathrm{f}$.

[3] Anonym. Die Substitution von ärztlichen Aufgaben durch die Pflege ist alternativlos. Rechtsdepesche 27.07.2019. Im Internet: (Stand: 11.08.2021) https://www.rechtsdepesche.de/unser-sys tem-wird-in-fuenf-jahren-nicht-mehr-wiederzuerkennen-sein/

[4] Großkopf V, Klein H. Recht in Medizin und Pflege. 5. Aufl. Balingen: Spitta; 2020. $212 \mathrm{f}$.

[5] Großkopf V. Streitpunkt i. v.-Injektion: Eine delegationsfähige Maßnahme? Die Schwester/Der Pfleger 2008; 11: $1064 f$. 
[6] Interview mit Prof. Dr. Volker Großkopf: Die Verantwortungsbereiche von Arzt und Pflegekraft, Rechtsdepesche.

(Stand: 29.09.2021) https://www.rechtsdepesche.de/ die-verantwortungsbereiche-von-arzt-undpflegekraft/

[7] SG Speyer vom 27.7.2005 - AZ: S 2 P 122/03 veröffentlicht in Großkopf V, Hrsg. Kompaktwissen Haftpflichtrecht; 2019; 2. Aufl. Köln: G \& S-Verlag $137 f f$.

[8] Großkopf V. Kompaktwissen Haftpflichtrecht; 2019; 2. Aufl. Köln: G \& S-Verlag 69f.

[9] OLG Dresden vom 24.07.2008, AZ: 4u1857/07 in Rechtsdepesche für das Gesundheitswesen. 2008. 240f.

[10] Bernd Schöneck: Zwischen rechtlicher Komfortzone und Alptraum, Rechtsdepesche. (Stand 29.09.2021) https://www. rechtsdepesche.de/zwischen-rechtlicherkomfortzone-undalptraum/

[11] Di Bella M. Delegation der Behandlungspflege. Rechtsdepesche für das Gesundheitswesen 1. Aufl. 2008. 29f.
Bibliografie

Phlebologie 2021; 50: 343-355

DOI 10.1055/a-1421-2625

ISSN 0939-978X

(c) 2021. Thieme. All rights reserved.

Georg Thieme Verlag KG, Rüdigerstraße 14

70469 Stuttgart, Germany 


\section{Punkte sammeln auf CME.thieme.de}

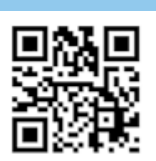

Diese Fortbildungseinheit ist in der Regel 12 Monate online für die Teilnahme verfügbar.

Den genauen Einsendeschluss finden Sie unter https://eref.thieme.de/cxgwmph.

Sollten Sie Fragen zur Online-Teilnahme haben, finden Sie unter https://cme.thieme.de/hilfe

eine ausführliche Anleitung. Wir wünschen viel Erfolg beim Beantworten

der Fragen!

Unter https://eref.thieme.de/cxgwmph oder über den QR-Code kommen Sie direkt zur Startseite des Wissenstests.

VNR 2760512021160214387

\section{Frage 1}

Was ist eine delegierbare Leistung?

A Eine Leistung, die die Arzthelferin machen sollte, aber der Arzt übernimmt.

B Eine Leistung, die ein Bundestagsabgeordneter vollzieht.

C Eine Beauftragung im Rahmen der Mitarbeiter-Selbstverwaltung.

D Eine ärztliche Leistung, die unter gewissen Bedingungen von seinen nichtärztlichen Mitarbeitenden durchgeführt werden kann.

E Eine abrechenbare Leistung, die der Arzt selbst durchführt.

\section{Frage 2}

Welche Kriterien sind für eine delegierbare Leistung nicht relevant?

A Die Leistung muss einfach durchzuführen sein.

B Der Arzt beherrscht die Leistung selbst nicht so gut wie die MFA (medizinische Fachangestellte).

$C$ Die MFA hat die Informationen rund um diese Leistung in der Ausbildung gelernt.

D Die MFA hat nachgewiesen, die Leistung zu beherrschen.

E Die Leistung birgt keine oder kaum Risiken.

\section{Frage 3}

Inwiefern ist das Qualitätsmanagement (QM) für die delegierbaren Leistungen relevant?

A Das Qualitätsmanagement erklärt den juristischen Rahmen, nach dem die Leistungen delegierbar sind.

B Im Qualitätsmanagement werden Arbeits- und Verfahrensanweisungen hinterlegt, sodass der Ablauf einer delegierbaren Leistung nachvollziehbar ist.

C Im QM sind die Arbeiten gelistet, die der Arzt nicht beherrscht.

D Das QM ist nicht relevant für das Thema delegierbare Leistungen.

E Im QM wird hinterlegt, welche Abrechnungsvorgaben gelten.

\section{Frage 4}

Was trifft in Bezug auf das Recht von medizinischen Fachangestellten (MFA), eine delegierbare Leistung abzulehnen, nicht zu?

A Die Durchführung einer delegierbaren Leistung kann abgelehnt werden, wenn man sich bei der Durchführung der delegierbaren Leistung nicht sicher fühlt.

B Die Durchführung einer delegierbaren Leistung kann abgelehnt werden, wenn die MFA der Auffassung ist, die Leistung ist bei dem entsprechenden Patienten nicht indiziert.

C Die Durchführung einer delegierbaren Leistung kann abgelehnt werden, wenn die delegierbare Leistung nicht im Ausbildungskatalog der MFA enthalten ist.

D Die Durchführung einer delegierbaren Leistung darf nicht abgelehnt werden.

E Die Durchführung einer delegierbaren Leistung kann abgelehnt werden, wenn sich die MFA an dem Tag gesundheitlich nicht gut fühlt.

\section{Frage 5}

Welche der folgenden Aussagen ist korrekt?

A Eine Blutentnahme darf ein Arzt unter keinen Umständen an eine MFA delegieren.

B Die Stellung der definitiven Diagnose darf ein Arzt unter keinen Umständen an eine MFA delegieren.

C Ein EKG zu schreiben, darf ein Arzt unter keinen Umständen an eine MFA delegieren.

D Blutdruckmessen darf ein Arzt nicht an eine MFA delegieren.

E Es gibt keine Leistung, die nach ausreichender Einweisung nicht delegierbar wäre.

- Weitere Fragen auf der folgenden Seite... 


\section{Punkte sammeln auf CME.thieme.de}

\section{Frage 6}

Welche juristischen Kriterien spielen bei delegierbaren Leistungen keine Rolle?
A die objektive Gefährlichkeit
B die materielle Qualifikation
$C$ die Ausbildungsordnung
D das Alter der MFA
E die Prüfungsordnung

\section{Frage 7}

Nur eine der folgenden Aussagen hinsichtlich der Delegation im Rahmen von Impfungen ist korrekt. Welche?

A Die Tätigkeit der MFA hat sich auf das Aufziehen der Spritze und die Hautdesinfektion zu beschränken.

B Die Patientenaufklärung kann die MFA nach entsprechender Schulung übernehmen.

C Mindestens eine MFA muss als Zeugin anwesend sein, wenn der Arzt die Impfung durchführt.

D Medizinische Fachangestellte erlernen die Verimpfung von entsprechenden Substanzen in ihrer Ausbildung.

E Ist die ärztliche Anordnung zur Verabreichung von Impfungen an eine MFA erstmals erfolgt, ist sie bis auf Weiteres für sämtliche in der Praxis verabreichten Impfstoffe gültig.

\section{Frage 8}

Was zählt nicht zu den Grundvoraussetzungen einer Delegation ärztlicher Tätigkeiten?

A Der Anweisende hat zuvor das Risiko der möglicherweise auftretenden Komplikationen zu prüfen.

B Der Anweisende hat zuvor den Schwierigkeitsgrad des Eingriffs abzuklären.

C Der Angewiesene hat die subjektiven Fähigkeiten des Anweisenden festzustellen.

D Zu prüfen sind vor allem die durch ein Ausbildungszeugnis bescheinigten Fähigkeiten und Kenntnisse des Angewiesenen.

E Der Angewiesene muss die subjektive Fähigkeit zur Ausführung der angewiesenen Tätigkeit besitzen.

\section{Frage 9}

Welche der folgenden Zuordnungen stimmt nicht?

A Die formelle Qualifikation entspricht den tatsächlichen Fähigkeiten.

B Die formelle Qualifikation entspricht dem, was in der Ausbildung erlernt wurde.

C Die materielle Qualifikation entspricht den tatsächlichen Fähigkeiten.

D Durchführungsverantwortung ist die Verantwortung des angewiesenen Fachpersonals, die angewiesene Maßnahme sach- und fachgerecht zu erledigen.

E Horizontale Arbeitsteilung entspricht der Aufteilung von Aufgaben innerhalb einer Berufsgruppe.

\section{Frage 10}

Was bedeutet der Begriff Remonstrationsrecht?

A kollektive Arbeitsverweigerung

B das Recht zur Zurückweisung angeordneter Aufgaben

C die Teilnahme an betriebsinternen Demonstrationen

D die Teilnahme an außerbetrieblichen Demonstrationen

E Durchführung der Maßnahme mit Widerspruchsvorbehalt 